Herangehensweise und die Prinzipien des vorsorgenden Wirtschaftens in einen gesellschaftlichen Gestaltungsprozeß eingehen.

\section{Literafur}

- Bennholdt-Thomsen, V./ Mies, M./Werlhof, C. von: Frauen, die letzte Kolonie, 3. Aufl., Zürich 1992.

- Biesecker, A.: Kooperation, Netzwerk, Selbstorganisation - Prinzipien für eine faire und vorsorgende Ökonomie, in: A. Biesecker und K. Grenzdörffer (Hg.): Kooperation, Netzwerk, Selbstorganisation - Elemente demokratischen Wirtschaftens, Pfaffenweiler: Centaurus 1996, S. 9-21.

- Biesecker, A.: Für eine vorsorgende Wirtschaftsweise notwendige (neue?) Institutionen, in: Diskussionskreis "Frau und Wissenschaft" (Hg.): Ökonomie weiterdenken! Auf dem Weg zu einer feministisch sensiblen Ökonomie, Frankfurt/M., Campus 1997 (im Erscheinen).

- Busch-Lüty, C.: Ökonomie als Lebenswissenschaft, in: C Busch-Lüty et al. (Hg.): Vorsorgendes Wirtschaften. Frauen auf dem Weg zu einer Ökonomie der Nachhaltigkeit, Politische Ökologie, Sonderheft 6, München 1994, S.12-17.

- Harding, S.: Feministische Wissenschaftstheorie. Zum Verhältnis von Wissenschaft und sozialem Geschlecht, Berlin: Argument 1990.

- Hofmeister, S.: Der "blinde Fleck" ist das Ganze. Anmerkungen zur Bedeutung der Reproduktion in der Ökonomie, in: K. Grenzdörffer u.a. (Hg.): Neue Bewertungen in der Ökonomie, Pfaffenweiler: Centaurus 1995, S. 5165.

- Jochimsen, M./Knobloch, U.: Making the hidden visible. The importance of caring activities and their principles for any economy, in: Special Issue: Women, Ecology and Economics, Ecological Economics 20 (1997), S. 107-112. - Jochimsen, M./ Knobloch, U./ Seidl, I.: Vorsorgendes Wirtschaften. Konturenskizze zu Inhalt und Methode einer ökologisch und sozial verträglichen Ökonomie, in: C. BuschLüty et al. (Hg.): Vorsorgendes Wirtschaften. S.0., S. 6-11. - Kaufmann-Hayoz, R.: Verantwortung aus Mitgefühl. Die Beziehung zur Natur als Basis für umweltverantwortliches Wirtschaften, in: C. Busch-Lüty et al. (Hg.): Vorsorgendes Wirtschaften. S.0., S. 36-39.

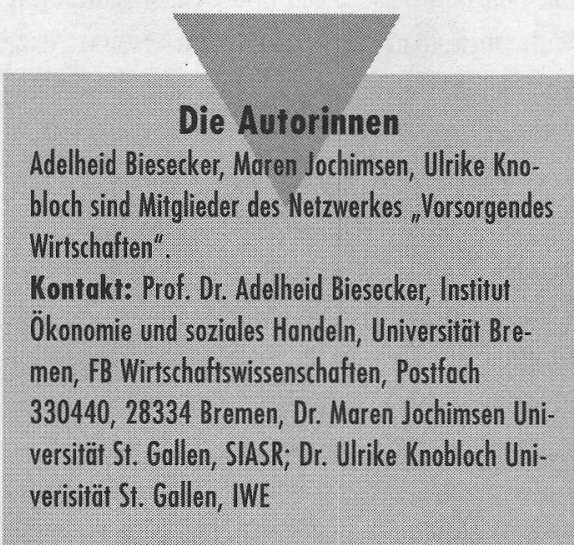

Eine Polemik gegen den Houshalt als Ausgangspunkt allgemeiner Richtlinien

\title{
Ökofeministisch wirtschaften?
}

\section{Die Durchsetzung ökonomischer Gleichberechtigung der Geschlechter ist vor- wiegend ein politökonomisches Problem. Neuartige ethische Fragen, auf die die Prinzipien der Hausarbeit eine Antwort geben müßten, spielen demgegenüber nur eine untergeordnete Rolle. Vielmehr geht es um die Frage, mittels welcher Bündnisse Ansätze zur Umsteverung durchgesetzł werden können.}

W Von Ulla Knapp erte haben wieder Konjunktur. Im wirklichen Leben die monetären, im wissenschaftlichen und politischen „Diskurs“ die ideellen. Wertebeschwörungen heben das Wohlbefinden. Man/frau kann politökonomischen Fach-Diskussionen aus dem Weg gehen. Worthülsen, in denen sich viele Wohlmeinende zuhause fühlen, lassen einen über die schwierige Aushandlung von Konflikten und Zahlungsbereitschaften hinwegschweben. Niemand fragt, wer die Deutsche Bank oder BMW dazu zwingen soll, ähnlich wie Lieschen Müller oder Otto Normalverbraucher ihre Steuern zu bezahlen, damit der Sozialstaat und die ökologische Umstrukturierung der Wirtschaft bezahlbar bleiben/werden. Es geht eben nicht um Verluste und Gewinne, sondern um Gut und Böse. Das gibt uns Frauen die Chance, auf der Sonnenseite zu stehen: der guten. Auf der anderen versammeln sich die Repräsentanten des „l'horreur économique" - früher hießen sie Patriarchat oder „Der Weiße Mann“(1), heute sind es die ,traditionellen Handlungsprinzipien des Wirtschaftens", ideell überhöht im neoliberalen Denken.

\section{Der neve Ansatz}

Neu ist, daß die ökofeministische Revolution, die Durchsetzung zukunftsfähiger Prinzipien des Umgangs mit Mensch und Natur, im wesentlichen im Kopfe stattfinden soll. Dabei sind Frauen - so die Gruppe ,Weiberwirtschaft" - objektiv wie subjektiv in besonderer Weise prädestiniert, diese mentale Umwälzung voranzutreiben.

„.... wenn wir von dem Teil unseres Lebens als Frauen ausgehen, den wir mit vorsorgenden Tätigkeiten verbringen, kommen wir $\mathrm{zu}$ den Prinzipien Vorsorgenden Wirtschaftens - gestaltet, strukturiert und erfahren durch Frauen, denn von hier aus bekommen wir wichtige Anregungen für Alternativen zur herrschenden Ökonomie. ... im Haushalt (können wir) ökonomi- sche Prinzipien finden, die sich grundsätzlich von den Handlungsprinzipien der freien Marktwirtschaft unterscheiden und uns einen Ausweg aus der derzeitigen Krisensituation zeigen können“ (2). Genannt werden: Vorsorge, Fehlerfreundlichkeit, Langsamkeit, Kooperation.

Mit der Beschreibung des Haushaltsgeschehens als Inkarnation des „Sorgens“, der Fürsorglichkeit, der Gebrauchswertorientierung wird $\mathbf{u}$. a. Bezug genommen auf die sozialökonomische Konzeption des Haushalts bei Adelheid Biesecker. $\mathrm{Ob}$ auch bei ihr die Haushaltsproduktion - zumindest als Wille und Vorstellung - im positiven Sinne als Gegenbild einer Wirtschaftsweise verstanden wird, die ihre eigenen Grundlagen verbraucht, ist allerdings nicht ganz klar (3).

Kommunitaristen wird häufig vorgeworfen, daß sie zwar an Gemeinschaft und Gemeinsinn appellieren, die Werte, die sie sich wünschen, aber nicht spezifizieren, und im unklaren bleibt, ob sie die Gemeinschaft der Kegelclubs, des KuKlux-Klans oder der katholischen Kirche propagieren. Diesen Vorwurf kann man ökofeministinnen nicht machen. Für sie ist die Quelle der anzustrebenden Normen der Haushalt, ihr politisches Subjekt die Frau. Vom Haushalt, also den Frauen aus, läßt sich die Gesellschaft gestalten, in der die verschiedenen Erscheinungsformen des Guten (frauen-, um- und drittweltfreundlich) auf einen gemeinsamen Nenner gebracht sind.

\section{Feministische Kritik}

Für die „Alt-Frauenbewegten“ eine bittere These! 30 Jahre wissenschaftliche und politische Auseinandersetzungen um Hausarbeit und konservativ-reaktionäre Familienpolitik haben offensichtlich nicht vermocht, Leerformeln und Idealbilder à la Riehl und Würmeling aus den Sehnsüchten der Töchter zu vertreiben. Zwar ist Hausarbeit eine diskriminierte und $z$. T. komplementär-gegenstrukturell zum Erwerbsleben 
angelegte Vergesellschaftungsform der Arbeit in (kapitalistischen) Industriegesellschaften. Daraus folgt aber keinesfalls, daß sie - ihre Dynamik, ihre Handlungs- und Organisationsprinzipien, ihre Ergebnisse - als Vorbild für zukunftsfähiges Wirtschaften angesehen werden könnten. Je nach Betrachtungsobjekt kann durchaus auch das Gegenteil zutreffen: Für die BRD etwa kann die nach wie vor dominante Ausprägung von Familienformen durch die modernisierte ständestaatlich-patriarchalische Versorgerehe charakterisiert werden, dem Grundpfeiler einer frauenfeindlichen, asozialen, familien- und bevölkerungspolitisch ineffizienten Geschlechterpolitik. In den so charakterisierten Familien werden, überwiegend von Frauen, in erheblichem Umfang wirtschaftliche Leistungen, insbesondere soziale Dienstleistungen, erbracht. Dabei haben sie mit einer Vielzahl von Problemen zu kämpfen (z. B. Abhängigkeit von anderen sozialen Systemen, wie Verkehr, Medien, Wirtschaft; Gewalt gegen Frauen und Kinder; hoher Beitrag zur Umweltverschmutzung insgesamt; geringer Grad an eigenständiger technisch-organisatorischer Innovation, vielfach suboptimale Betriebsgröße; ungerechte, möglicherweise auch ineffiziente Arbeits-, Einkommens- und Machtverteilung). Diese Probleme und Leistungen, einschließlich der dazugehörigen familiären Handlungs- und Strukturprinzipien, sind vielfältig untersucht worden. Wer sich für ein Thema aus dem Teilbereich „Ökologische Effizienz der Hausarbeit und Geschlechtergleichheit" interessiert, sollte die Analyse auf dieser Grundlage in Angriff nehmen.

Unabhängig davon, wie man/frau den Haushalt politisch oder analytisch einordnet - eines stellt er allerdings in keinem Fall bereit: allgemeine Richtlinien wirtschaftlicher und politischer Koordination, speziell Handlungsregeln ökologischen Umsteuerns. Dazu tragen Prinzipien der Haushaltsproduktion - was immer darunter im einzelnen verstanden werde - allenfalls Wünsche, Metaphern und Sehnsüchte bei.

\section{- Ein geeignetes Koordinierungs- instrument?}

Solche Sehnsüchte - Kinderträume von der Guten Mutter, die fürsorglich und im wohlverstandenen Interesse aller zu handeln sucht - hegen wir alle. Faktisch ist schon unser Verhältnis zur wirklichen Mutter oft bestenfalls ambivalent (also nicht unbedingt Konfliktlösungs- und Koordinierungsvorbild). Was aber wichtiger ist:
Ganz grundsätzlich, unabhängig von den handelnden Personen, kann die Verhandlungs- oder Einfuihlungssituation im familiären Binnenraum kein Paradigma politökonomischer Koordinierung in modernen, hocharbeitsteiligen Gesellschaften sein. Wenn man die Abnehmer einer Ware nicht kennt, fehlt ohne gesellschaftiche Koordinierungsinstrumente einzelwirtschaftlicher Planungen schon die Information über das, was, wie, wo, wann und für wen produziert werden soll. Noch schwieriger ist die Frage zu lösen, wie die ProduzentInnen motiviert werden sollen, sich an die Wünsche der KonsumentInnen (oder auch an andere Zwecke) anzupassen. Märkte und andere Koordinierungsformen, mittels derer ökonomische Interessen in ein gesellschaftliches Abstimmungsverfahren eingebracht werden, lassen sich weder durch Appelle an „Fürsorglichkeit" verdrängen noch durch Prinzipien der Haushaltsproduktion (was immer das sei) ersetzen.

Schenkt man den Neoliberalen Glauben, so sind solche ,andere Koordinierungsformen“ ohnehin unnötig. Ökonomisch gesehen, beschere der Markt uns die beste aller Welten, wenn nur der Staat und die Gewerkschaften sich nicht immer wieder aufs Unheilvollste einmischen würden. Die Weiberwirtschaft äußert sich zu solch kollektiven Regelungen und Institutionen, die die Menschenfeindlichkeit des Marktes bislang in Grenzen gehalten haben, erst gar nicht. Ebenso wie die Manchester-Liberalen scheinen sie Staatsinterventionen nicht zu mögen, ohne solche Staatsverdrossenheit allerdings überhaupt einer Erwähnung (geschweige denn Begründung) für würdig zu erachten. Die ,alten“ Ökofeministinnen, wie Maria Mies oder Claudia v. Werlhof, formulierten da offener: „Der Bedarf an Substistenzsicherheit wird nicht durch das Vertrauen in das eigene Bankkonto oder den Sozialstaat gedeckt, sondern durch das Vertrauen in die Zuverlässigkeit der eigenen Gemeinschaft"(4). Was bleibt der Ökofeministin gegen den Neoliberalen? Die Ethik, der Haushalt, die gute (Haus)Frau, die Gemeinschaft der Frauen.

Ein solcher Appell an weiblichen Gemeinsinn für sorgendes Wirtschaften - ist notwendig, wo Sozial- und Umweltpolitik nicht (mehr) stattfinden. Viel ausgeprägter als bei uns ist dies in den USA der Fall. Vereinzelte Betriebskindergärten und ein hinreichendes Angebot an working poor (,nannies“) braucht man, wo die gesellschaftliche Solidarität in Form von Steuerzah- lungen nicht ausreicht, um eine angemessene und kostenfreie/günstige öffentliche Kinderbetreuung bereitzustellen. Was in den USA unter dem Stichwort „The Moral Dimensions of Economics" rangiert, ist oftmals nicht mehr als der Versuch, einen rudimentären Sozialstaat durch Appelle an Gemeinschaftswerte und Spendenfreude über seine brutalsten Auswüchse hinwegzuretten. Auch Plädoyers für die Stärkung des sog. Dritten - gemeinwirtschaftlichen - Sektors helfen über die Notwendigkeit sozialpolitischer Konsensfindung nicht hinweg. Selbst wenn man der Ansicht ist, dieser sei besser als der staatliche geeignet, (soziale) Dienstleistungen anzubieten, muß man eine angemessene Finanzierung, sprich: Subventionierung aus Steuern und Abgaben, sicherstellen, andernfalls befürwortet man schlicht Sozialabbau auf US-amerikanisches Niveau.

\section{Fraveninteressen}

In Europa geht es vielen demgegenüber glücklicherweise immer noch darum, den Wohlfahrtsstaat frauenfreundlich umzustrukturieren, was - solange man auch die weibliche Hälfte der unteren Einkommensschichten im Blick hat einschließt, seine Demontage zu verhindern. Fraglich ist allerdings, ob es ein verallgemeinerungsfähiges Fraueninteresse in dieser Frage gibt. Schon wenn man die Betrachtung auf entwickelte Industrieländer beschränkt, ist offensichtlich, daß Frauen unterschiedliche ökonomische und soziale Lagen und Orientierungen haben.

Meine These ist, daß eine an der Gesamtheit des weiblichen Geschlechts orientierte Politik wirtschaftlicher Gleichberechtigung - dazu gehören die eigenständige ökonomische Sicherung von befristet und teilzeitig Hausarbeit Leistenden, der Ausbau der Betreuungsinfrastruktur und die Gleichstellung im Beruf - vor allem Bündnisse mit den Gewerkschaften und eine sozialliberale Wohlfahrtsstaatlichkeit erfordern. Kleine Netze und (mehr oder weniger autoritäre) Gemeinschaften genießen demgegenüber da Priorität, wo professionelle Sozialpolitik als nicht bezahlbar gilt. Für Frauen aus mittleren und gehobenen sozialen Schichten mögen derartige Amerikanisierungsstrategien relativ mehr Vorteile bringen als die durch Sozial- und Tarifpolitik angestrebte Absicherung der Fraueneinkommen nach unten, für die anderen jedoch nicht. Die zentralen Fragen, von denen der Ökofeminismus ablenkt, lauten folglich (nach wie vor): 
Durch welche kollektiven Regelungen können die Prinzipien des ,guten Wirtschaftens" da durchgesetzt werden, wo der Markt versagt oder nur eingeschränkt funktioniert? Mittels welcher Bündnisse können Konzepte für eine Politik des Umsteuerns, eine frauen- und/oder umweltfreundliche Neustrukturierung der Wirtschaft, realisiert werden? Wie können Konflikte innerhalb gleichstellungs-, umwelt- und entwicklungspolitischer Zielbündel austariert werden? Wie läßt sich die damit verbundene Notwendigkeit, in erheblichem Umfang Einkommen, Vermögen und ökonomische Entscheidungsmacht umzuverteilen, durchsetzen?

Wenn es um Fraueninteressen im Sinne der wirtschaftlichen Gleichstellung geht, landen wir bei der Beschäftigungs-, Tarif- und Sozialpolitik. Meist wird verkannt, daß die Beschäftigungskrise in Deutschland nicht zuletzt das Resultat eines Strukturwandels der Geschlechterverhältnisse ist, an die wirtschaftliche und politische Verhältnisse nur rudimentär angepaßt wurden. M. a. W.: Weil wir in Deutschland West nicht mehr die (niedrige) Frauenerwerbsquote und die (hohen) Geburtenraten der 60er Jahre haben und weil die Anpassung des ostdeutschen an das westdeutsche System die der ostdeutschen an die westdeutsche Frauenerwerbsquote notwendig einschließt, gibt es das Problem der Erwerbslosigkeit und Sozialstaatskrise im heutigen Umfang. Politische Aufgabe der letzten 25 Jahre wäre es gewesen, staatliche Maßnahmen (Steuerpolitik, Sozialpolitik) und Tarifstrukturen (Löhne und Arbeitzeiten) im Sinne des neuen Geschlechterverhältnisses neu zu gestalten. Dies ist nur ansatzweise geschehen. Die Konsequenz - Massenerwerbslosigkeit - erschwert nun die Umstrukturierungs-, und d.h. vor allem: Umverteilungsprozesse in Richtung auf eine wirtschaftliche Gleichberechtigung der Geschlechter erst recht, ebenso wie sie die Chancen für ökologisches Umsteuern minimiert.

Ziel muß es deshalb heute sein, Wege aus der Beschäftigungs- und Sozialstaatskrise zu suchen, die zugleich den ökologischen Umbau und gleichstellungspolitische Reformen vorantreiben. Was die Analyse angeht, müßte der Schwerpunkt bei Fragestellungen liegen, die ausgehend von der Einsicht, daß politökonomische Ziele/Interessen normalerweise in einem konfliktären Verhältnis zueinander stehen, feministische und ökologische Ziele gleichwohl miteinander zu verknüpfen suchen, z. B.: Wie wirken sich umweltpolitische Maßnahmen (z. B.
Ökosteuer, ökologische Regionalentwicklung) auf die Erwerbssituation von Frauen aus? Welche Anforderungen implizieren umweltpolitische Maßnahmen für die Hausarbeit? Wie lassen sich die Mittelkonkurrenzen zwischen Sozial-/ Beschäftigungspolitik einerseits und Umweltpolitik andererseits auflösen/mildern? Wie sieht eine frauen- und umweltverträgliche Infrastrukturpolitik aus? Sind ökologisch sinnvolle neue Formen der Arbeits(zeit/ort)organisation auch gleichstellungspolitisch positiv zu bewerten?

\section{- Politische Spielräume}

Die Durchsetzung ökonomischer Gleichberechtigung der Geschlechter ist ein politökonomisches Problem. Neuartige ethische Fragen, auf die die Prinzipien der Hausarbeit eine Antwort geben müßten, spielen für ein frauenpolitisches Revival demgegenüber keine Rolle. Die Menschenrechte bieten hier nach wie vor eine brauchbare Leitschnur. Danach gilt: Menschen, auch Frauen, müssen einklagbare und gesellschaftlich verbürgte Rechte haben - auf eigenständige Existenzsicherung auch in Zeiten, in denen sie nicht zu Markte gehen können; auf professionelle Hilfe bei Krankheit und Pflegebedürftigkeit, auf Bildung und Ausbildung, auf politische Partizipation im Sinne der Formulierung und Vertretung ihrer eigenen Interessen, auf gleichberechtigte Teilnahme an Erwerbsarbeit und gesellschaftlichen Konsensfindungsprozessen.

Prinzipien der Hausarbeit liefern zur Formulierung dieser Menschenrechte keinerlei Beitrag. Umgekehrt wird ein Schuh daraus: Gemeinschaft, erwachsene Liebe und Ehe setzen im Prinzip gleiche Ressourcen voraus, so daß ich verhandeln und im Zweifelsfall gehen kann. Hinter diese politischen, wirtschaftlichen und sozialen Menschenrechte, die ohne Arbeitsteilung und Kooperation, ohne soziale Differenzierung und Integration, ohne Märkte und deren politische Koordinierung, ohne Individualisierung und Verhandlungswille und -fähigkeit nicht denkbar sind, darf kein politischer Weg zurückführen, kein ökofeministischer und kein kommunitaristischer.

Menschenrechte für Frauen einzufordern heißt, die gesamtgesellschaftlich und makroökonomisch nachweisbare Hierarchie der Geschlechter abzubauen. Diese Hierarchie in Form geschlechtsspezifischer Charaktereigenschaften, Verhaltensweisen oder Einstellungen auf die sozialpsychologische oder -philosophische Ebe-

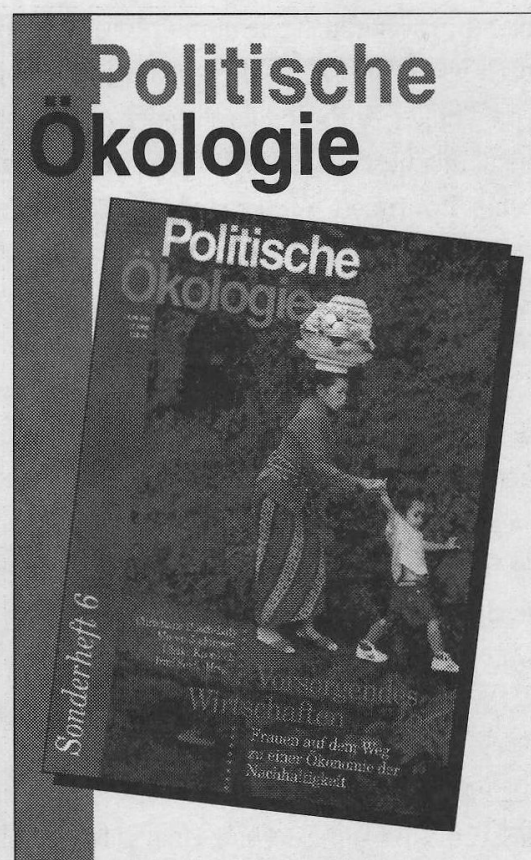

Vorsorgendes Wirtschaften

Frauen auf dem Weg zu einer Ökonomie der Nachhaltigkeit Politische Ökologie, Sonderheft 6

Frauen bringen durch ihre Arbeit im familiären Bereich einen Erfahrungshintergrund mit, der in der Diskussion über nachhaltiges Wirtschaften bislang vernachlässigt wurde: die Orientierung am Lebensnotwendigen, das Vorsorgeprinzip und die Kooperation.

In dem ausschließlich von Frauen konzipierten und erarbeiteten Heft werden erstmals Ansätze und Erfahrungen zum Vorsorgenden Wirtschaften zusammengetragen und deren ökologische und menschengerechte Bedeutung skizziert:

M. Jochimsen, U. Knobloch und I. Seidl: Vorsorgendes Wirtschaften eine Konturenskizze

- C. Busch-Lüty: Das Nachhaltigkeitsprinzip als wissenschaftstheoretische Herausforderung

- M. Mies: Brauchen wir eine neue "Moral Economy"

- J. Davis und G. Kocsis:

Kann Ökononomie weiblich sein?

- A. Zahrnt: Die politischen Rahmenbedingungen für Vorsorgendes Wirtschaften

H. Saibold: Gesellschaftliches Engagement und privates Konsumverhalten

Jetzt für DM 14,80 ökom GmbH überall in der Waltherstr.29, Rgb.

Bahnhofspresse 80337 München

erhältlich oder direkt Tel.: 089/544 184-0 bestellen bei: Fax: 089/544 184-99 
ne $\mathrm{zu}$ projizieren, verfehlt allerdings - theoretisch wie empirisch - die Verhaltensrepertoires von Frauen. Insbesondere eignen sich Frauen nicht qua Geschlecht zur Durchsetzung ökologischer Prinzipien. Weder sind sie die besseren, noch die aufgrund ihrer Fürsorglichkeit ökologisch und entwicklungspolitisch engagierteren Menschen. Das Denken in dualistischen Geschlechterkonstruktionen sollte frau Stammtischen überlassen - egal ob diese den Frauen nette oder weniger nette Eigenschaften unterstellen. Um ethisch als gleiche anerkannt zu werden, bedürfen sie ebensowenig wie Juden und Schwarze eines besonderen Leistungsnachweises; es genügt, daß sie Menschen sind.

Als Menschen können sie an einer Politik der Nachhaltigkeit und an der Bekämpfung der Armut in der Welt interessiert sein. Die Verhältnisse, die der Durchsetzung einer solchen Politik entgegenstehen, mögen unüberwindlich scheinen. Es kommt aber darauf an, dies zu ändern. Ökofeminismus und der horreur économique helfen dabei nicht weiter. Als alter ego des Neoliberalismus lassen sie sich treffender charakterisieren denn als seine Alternative.

\section{Anmerkungen}

(1) Vergleiche die sogenannten "Bielefelderinnen", C. v. Werlhof, V. Bennholdt-Thomsen, M. Mies.

(2) Weiberwirtschaft. Frauen - Ökonomie - Ethik. Mit Beiträgen von H. Bernhard Filli, A. Günter, M. Jochimsen, U. Knobloch u.a., Luzern 1994, S. 38, S. 40.

(3) Vgl. z.B. Biesecker, A.: Lebensweltliche Erneuerung der Ökonomie - über alte und neve Formen weiblichen Wirtschaftens, in: Zeitschrift für Frauenforschung, 14. Jg., Heft 3/1996, S. $102 \cdot 116$

(4) Mies, M. und V. Shiva: Ökofeminimus. Beiträge zu Praxis und Theorie, Zürich 1994, S. 415
Dieser Beitrag ist die stark gekürzte Fassung eines Aufsates, der erscheint in: August, U. (Hg.): Weiberwirtschaft, Ökofeminismus und Konsumboykott (Arbeitstitel), Sozialwissenschaftliches Institut der EKiD, Bochum 1997 (Kontakt: Evangelische Akademie Iserlohn); Literatur siehe ausführlich dort.

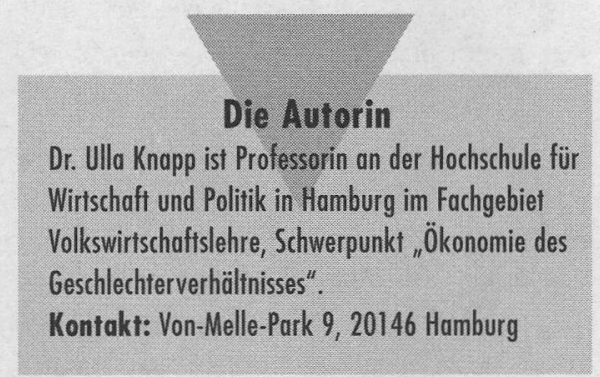

\section{Literatur zum Spezial}

- Agarwal, Bina (1989): Neither Sustenance nor Sustainability: Agricultural Strategies, Ecological Degradation and Indian Women in Poverty, in: Agarwal, Bina (Hrsg.): Structures of Patriarchy: The State, the Community and the Household, Zed Books, S. 83-120

- Agarwal, Bina (1992): The Gender And Environment Debate: Lessons From India, in: Feminist Studies, 18, S. 119-158

- Agarwal, Bina (1994): A Field of One's Own: Gender and Land Rights in South Asio, Cambridge University Press

- Agarwal, B. (1997): „Bargaining“ and Gender. Relations: Within and beyond the Household. In: Feminist Economics, 1, 1.50

- Bakker, I. (Hg.) (1994): The Strategic Silence: Gender and Economic Policy, London

- Biesecker, Adelheid/Wolf, Sabine (1995): Ökonomie und Geschlechterverhälinis; Stand der Diskussion und Theorieskizze. In: Priddat, Birger P./Seifert, Eberhard K. (Hrsg.): Neuorientierungen der ökonomischen Theorie; Zur moralischen, institutionellen und evolutorischen Dimension des Wirtschuffens, Marburg, S. 123-152

- Braidotti, Rosi, et al. (1994): Women, the Environment and Sustainable Development. Towards a Theoretical Synthesis, Zed Books

- Busch-Lüty, Christiane, et al. (Hisg.) (1994): Vorsorgendes Wirtschaften. Frauen auf dem Weg zu einer Ökonomie der Nachhaltigkeit, Politische Ökologie

\section{Sonderheft 6}

- Dankelman, I./Davidson, J. (1988): Women and the Environment in the Third World, London

- Ecological Economics 1997, Vol 20, Nr. 2: Special Issue: Women, Ecology and Economics

- Folbre, N. (1994): Who Pays for the Kids? Gender and the Structure of Constraint, London

- Harcourt, Wendy (Hrsg.) (1994): Feminist Perspectives on Sustainable Development, Zed Books

- Jacobsen, Joyce, P. (1994): The Economics of Gender, Blackwell

- Kabeer, Noila (1994): Reversed Realities. Gender Hierarchies and Development Thoughts, London/New York

- Kuiper, Edith/Sap, Jolande (Hrsg.) (1995): Out Of

The Margin. Feminist Perspectives on Economics, London

- Merchant, C. (1995): Earthcare: Women and the Environment, London/New York

- Mies, M./Shivo, V. (1993): Ecofeminism, London

- Nelson, Julie A. (1995): Feminism and

Economics, in: The Journal of Economic Perspective, Nr. 2, S. $131 \cdot 148$

- Nelson, Julie/Ferber, Marianne (Hrsg.) (1993):

Beyond Economic Man, University of Chicago Press
- Regenhard, Ulla/Maier, Friederike/Carl, Andrea-Hilla (Hrsg.) (1994): Ökonomische Theorien und Geschlechterverhälınis. Der männliche Blick der Wirtschaftswissenschaft, Fachhochschule für Wirtschaft, Berlin

- Schultz, Irmgard (Hrsg.) (1993): GlobalHaushalt. Globalisierung von Stoffströmen - Feminisierung von Verantwortung, Frankfurt

- Schultz, Irmgard/Weller, Ines (Hirg) (1995): Gender \& Environment: Ökologie und die Gestallungsmacht der Frouen, Frankfurt

- Sen, Gita/Grown, Caren (1988): Development, Crises and Alternative Visions. Third World Women's Perspective, London

- Shiva, Vandana (1989): Das Geschlecht des Lebens. Frauen, Ökologie und Dritte Welt, 1. dt. Auflage, Rotbuch Verlog

- Sontheimer, Sally (Hrsg.) (1991): Women and the Environment: A Reader: Crisis and Development in the Third World, London

- Wichterich, Christo (1992): Die Erde bemuttern. Frauen und Ökologie nach dem Erdgipfel in Rio, HeinrichBöll-Stiftung (Hrsg.)

- World Development (1995), Vol 23, Nr. 11: Special Issue: Gender, Adjustment and Macroeconomics - Zein-Elabdin, E. (1996): Development, Gender, and the Environment. Theoretical or Contextual 
(c) 20I0 Authors; licensee IÖW and oekom verlag. This is an article distributed under the terms of the Creative Commons Attribution Non-Commercial No Derivates License (http://creativecommons.org/licenses/by-nc-nd/3.o/), which permits unrestricted use, distribution, and reproduction in any medium, provided the original work is properly cited. 